\title{
Estudio sobre la interrelación entre la dislalia y la personalidad del niño
}

\author{
Juan Manuel Moreno Manso ${ }^{1}$ \\ María Rosa Mateos García ${ }^{2}$
}

${ }^{1}$ Dpto. de Psicología y Sociología de la Educación, Universidad de Extremadura, Badajoz

${ }^{2}$ Colegio Público Nuestra Señora de Fátima, Carcaboso (Cáceres)

España

althea@correo.cop.es 


\section{Resumen}

Introducción. En este artículo analizamos la existencia de características de la personalidad asociadas a la dislalia infantil. Las referencias bibliográficas relativas al tema son escasas y poco recientes. Diferentes autores señalan que determinados rasgos de la personalidad pueden actuar en unas ocasiones como desencadenantes y/o favorecedores de dichas alteraciones y en otros casos como mantenedores y/o agravantes de la dificultad del niño.

Método. El estudio que presentamos a continuación, refleja los resultados de la aplicación del Cuestionario de Personalidad para Niños (ESPQ) y del Cuestionario de Ansiedad Infantil (CAS) a una muestra de 20 niños diagnosticados con dislalia, de edades comprendidas entre los 6 y 8 años.

Resultados. Los datos corroboran que los niños con dislalia manifiestan una ansiedad, tensión e inseguridad superior a la media y son más propensos a perder el control emocional. Asimismo, se muestran inhibidos y tienen dificultades para relacionarse con los demás. Por otra parte, constatamos la dificultad existente a la hora de delimitar si los rasgos de personalidad de estos niños causan el desorden lingüístico, o en cambio son un efecto del mismo.

Discusión. La investigación destaca la necesidad de un mayor conocimiento y profundización sobre el papel que desempeña la personalidad en los trastornos del lenguaje de mayor incidencia durante las etapas escolares iniciales. Delimitar las características de la personalidad del niño con dislalia, nos permitirá adaptarnos a su realidad durante el proceso de intervención. Debemos tener muy presentes los aspectos personales, relacionales, sociales y emocionales del niño, dado que el conocimiento de los mismos, puede facilitar la superación más rápida de las dificultades que presenta.

Palabras Clave: dislalia, investigación, personalidad, trastorno del habla 


\section{Introducción}

Con este trabajo llevamos a cabo una investigación sobre las características de la personalidad asociadas a una de las patologías del lenguaje oral que tienen una mayor incidencia en Educación Infantil y Primaria. Concretamente nos referirnos a la dislalia infantil. Es lógico pensar que determinadas características de la personalidad de un niño puedan repercutir en cierta medida en sus repertorios lingüísticos y comunicativos. Por tanto, la finalidad básica de esta investigación es conocer las tendencias de personalidad de sujetos con dislalias.

Son numerosos los acontecimientos que pueden repercutir en el lenguaje infantil. La dificultad para interaccionar con otros niños, un ambiente familiar autoritario o poco estimulante, baja autoestima, celos fraternales, dificultades escolares, rechazo por parte de los compañeros, falta de aceptación personal, falta de afecto paterno, sobreprotección, conflictividad escolar, ausencia de padres, tensiones en el núcleo familiar, actitudes ansiosas parentales, etc., pueden perturbar la personalidad de un niño. Estos sucesos pueden provocar en el niño: inhibición, conductas de evitación, aislamiento, agresividad, ansiedad, frustración, timidez, reducción de contacto con otras personas, sentimientos de inferioridad, mutismo, inseguridad, pérdida de confianza en sí mismo y desajustes emocionales, abocándolo hacia la aparición de una patología del habla.

Cuando profundizamos a nivel teórico en los trastornos del lenguaje constatamos escasas referencias bibliográficas relativas al tema, siendo la mayoría de ellas poco recientes. De manera generalizada, diferentes autores plantean que determinadas características personales pueden desempeñar un papel relevante en la aparición y en el mantenimiento de la dislalia. De ahí que pensemos que la valoración de las características de la personalidad del niño debe formar parte, de una manera indispensable, del proceso de exploración e intervención ante la detección de este desorden del habla (Cano y Navarro, 2003; Moreno, 2004).

Una de las primeras referencias al tema es la de Solomon en 1961. Según Solomon los niños con dislalia se diferencian de los niños con un desarrollo normal del habla en sus relaciones fraternales y en sus tensiones, angustias y miedos. Años más tarde, Sussman (1975) 
considera que la dificultad articulatoria infantil puede estar ocasionada por la inmadurez del niño, bloqueos emocionales, conductas inestables, etc. Waller, Sollod, Sander y Kunkicki (1983) señalan la incidencia de factores psicoafectivos en los niños con dislalia. En 1990, Perelló mantiene que la dislalia es más frecuente en niños con determinadas características de la personalidad. Según él, estos niños durante la exploración se muestran intranquilos, inconstantes, distraídos, tímidos, a veces apáticos y faltos de interés por aprender. Asimismo, plantea que las madres de estos niños son más neuróticas y altivas que las madres de los niños con un desarrollo normal del habla. Aguilar (1991) incluye dentro de los trastornos del lenguaje como efecto de las condiciones de aprendizaje, personalidad, motivación y situación, las dislalias múltiples funcionales. Ajuriaguerra y Marcelli (1982), Serón y Aguilar (1992), Ramos y Manga (1996), Busto (1998) y García-Valdecasas (2000) consideran que existe una estrecha relación entre trastornos psicoafectivos en la primera infancia y perturbaciones en el habla. Y Bruno y Sánchez, en una publicación de 1994 plantean que entre los factores favorecedores de la aparición y/o mantenimiento de la dislalia se encuentran las presuntas características psicopatológicas del niño. Es decir, que la personalidad del niño podría actuar como un factor etiológico indirecto en la alteración articulatoria.

Massana y Artal (1997) señalan que entre las causas que pueden motivar la dislalia se encuentran características psicológicas tales como la falta de aceptación del niño, la baja autoestima y las perturbaciones afectivas y emocionales.

En esta línea de investigación, y tras comprobar la escasez de estudios previos sobre los rasgos de la personalidad de los niños con dislalia, evaluamos estas características en 20 niños diagnosticados de dislalia, utilizando para ello dos pruebas: el Cuestionario de Personalidad para niños E.S.P.Q., que valora la personalidad a través de la baremación de distintos factores duales; y el Cuestionario de Ansiedad Infantil C.A.S., que indica el nivel de ansiedad generalizado que experimenta el sujeto. Tratamos de identificar los rasgos comunes de personalidad en los niños que presentan dislalia, contribuyendo con ello a un mayor conocimiento de la patología del habla de mayor incidencia en la población escolar.

Las hipótesis que pretendemos verificar son las siguientes: 1) los niños con dislalia se muestran inhibidos y tienen dificultades para relacionarse con los demás; 2) los niños con 
dislalia manifiestan una ansiedad, tensión e inseguridad superior a la media y son más propensos a perder el control emocional.

\section{Método}

\section{Sujetos}

La población objeto de estudio son 20 alumnos procedentes de centros escolares públicos ubicados en la provincia de Cáceres. Todos los alumnos seleccionados tienen edades comprendidas entre los 6 y 8 años, se encuentran escolarizados en Educación Primaria, tienen informe del Equipo de Orientación Psicopedagógica y reciben sesiones de logopedia en el centro.

Tabla 1. Distribución de los niños según el sexo y la edad.

\begin{tabular}{|c|c|c|c|c|}
\hline & $\mathbf{6}$ años & $\mathbf{7}$ años & $\mathbf{8}$ años & N \\
\hline VARÓN & 10 & 2 & 2 & 14 \\
\hline MUJER & 4 & 2 & - & 6 \\
\hline TOTAL & 14 & 4 & 2 & 20 \\
\hline
\end{tabular}

En la tabla 1 vemos como el $70 \%$ de los niños con dislalia tienen una edad cronológica de 6 años. Asimismo en la muestra constatamos la mayor incidencia del varón sobre la mujer. El $70 \%$ de los niños con dislalia son varones y el 30\% mujeres.

\section{Instrumentos}

Los instrumentos utilizados para la evaluación de las características de la personalidad de los niños con dislalia, teniendo en cuenta la edad de los menores, han sido el Cuestionario de Personalidad para niños (ESPQ) y el Cuestionario de Ansiedad Infantil (CAS).

\section{Procedimiento}

En primer lugar utilizamos un cuestionario con el objeto de evaluar la personalidad de los niños. El Cuestionario de Personalidad para niños - ESPQ (Coan y Cattell, 2002) es uno de los instrumentos más adecuados y convenientes para evaluar la personalidad de los niños en edad escolar. Permite la apreciación de trece rasgos de primer orden y dos de segundo en 
niños de 6 a 8 años. La prueba se divide en dos partes: A1 y A2 que exigen entre 30 y 40 minutos, según el grado de maduración de los niños. Cada una contiene 80 cuestiones (seis en cada uno de los doce rasgos de personalidad y ocho para el aspecto intelectual). Los factores de primer orden evaluados son: reservado - abierto, inteligencia baja - inteligencia alta, afectado por los sentimientos - emocionalmente estable, calmoso - excitable, sumiso - dominante, sobrio - entusiasta, despreocupado - consciente, cohibido - emprendedor, sensibilidad dura sensibilidad blanda, seguro - dubitativo, sencillo - astuto, sereno - aprensivo y relajado tenso. Además permite la cuantificación de dos factores de segundo orden: ajuste - ansiedad e introversión - extroversión.

En un segundo momento, dados los resultados obtenidos por los niños en el factor de ansiedad de segundo orden del ESPQ, decidimos aplicar el Cuestionario de Ansiedad Infantil - CAS (Gillis, 1999). Es una prueba idónea para evaluar la ansiedad infantil en niños de 6 a 8 años. El CAS permite valorar la ansiedad generalizada del niño, a partir del decatipo y la puntuación centil que se obtiene. Constituye una derivación, por su estructura y formato, de los cuestionarios de Personalidad de Cattell, como el ESPQ, con el que correlaciona en su factor de ansiedad de segundo orden.

\section{Análisis Estadístico}

Para realizar el análisis de los datos obtenidos utilizamos estadísticos descriptivos, con la finalidad de establecer la frecuencia de los factores analizados en la muestra objeto de estudio.

\section{Resultados}

A) Resultados del Cuestionario de Personalidad para niños (ESPQ)

Antes de comenzar a describir las tablas correspondientes a la aplicación del Cuestionario de Personalidad para niños (ESPQ), es conveniente aclarar que los decatipos 5 y 6 son valores medios, 4 y 7 muestran una pequeña desviación (en una y otra dirección, respectivamente). 2 - 3 y 8 - 9 indican una gran desviación, y 1 y 10 son valores extremos, entendiendo todas estas posiciones como relativas a la población específica sobre la cual se realizó la tipificación. 
A continuación en la tabla 2, vemos los resultados de la aplicación del Cuestionario de Personalidad para niños ESPQ en los Factores de Primer Orden.

Tabla 2. Resultados del Cuestionario de Personalidad ESPQ en los Factores de Primer Orden (1)

\begin{tabular}{|r|r|r|r|r|r|r|r|}
\hline DECATIPOS & Factor 1 & Factor 2 & Factor 3 & Factor 4 & Factor 5 & Factor 6 & Factor 7 \\
\hline 1 & - & 2 & - & - & 2 & - & 2 \\
\hline 2 & - & 2 & - & 2 & 4 & - & - \\
\hline 3 & 8 & - & 12 & 2 & 4 & 12 & 6 \\
\hline 4 & 4 & 4 & - & 2 & 2 & 2 & 2 \\
\hline 5 & 4 & 6 & 4 & - & - & 2 & 4 \\
\hline 6 & 2 & 2 & 4 & 6 & 2 & 4 & 4 \\
\hline 7 & 2 & - & - & 4 & - & - & 2 \\
\hline 8 & - & 2 & - & 2 & 4 & - & - \\
\hline 9 & - & 2 & - & 2 & 2 & - & - \\
\hline 10 & - & - & - & - & - & - & - \\
\hline Total & 20 & 20 & 20 & 20 & 20 & 20 & 20 \\
\hline
\end{tabular}

Factor 1: Reservado-Abierto Factor 2: Inteligencia alta-baja Factor 3: Emocionalmente afectado-Estable

Factor 4: Calmoso-Excitable Factor 5: Sumiso-Dominante Factor 6: Sobrio-Entusiasta Factor 7: Despreocupado-Consciente

\begin{tabular}{|lcccccccc|}
\hline & Media & Sx & Mo & Var & \multicolumn{3}{c|}{ Percentiles } \\
& & & & & 25 & 50 (Mdn) & 75 \\
\hline Reservado-abierto & 4,300 & 1,418 & 3,0 & 2,011 & 3,000 & 4,000 & 5,250 \\
Inteligencia baja-alta & 4,900 & 2,424 & 5,0 & 5,878 & 3,500 & 5,000 & 6,500 \\
Emocional afectado-estable & 4,000 & 1,333 & 3,0 & 1,778 & 3,000 & 3,000 & 5,250 \\
Calmoso-excitable & 5,800 & 2,201 & 6,0 & 4,844 & 3,750 & 6,000 & 7,250 \\
Sumiso-dominante & 4,600 & 2,914 & 2,0 & 8,489 & 2,000 & 3,500 & 8,000 \\
Sobrio-entusiasta & 3,900 & 1,287 & 3,0 & 1,656 & 3,000 & 3,000 & 5,250 \\
Despreocupado-consciente & 4,300 & 1,829 & 3,0 & 3,344 & 3,000 & 4,500 & 6,000 \\
\hline
\end{tabular}

En la distribución de los casos en el Factor 1 (Reservado - Abierto) en la muestra analizada en nuestra investigación, constatamos que un $60 \%$ de los niños se muestran reservados en las interacciones sociales, alejándose del contacto con compañeros (el 40\% de los niños 
manifiestan una gran desviación y en el $20 \%$ restante la desviación en este factor de personalidad es pequeña, respectivamente). Los resultados no son significativos, puesto que el $30 \%$ de los niños se sitúan en valores medios. Y por último el 10\% restante se muestran abiertos, afectuosos, participativos y sociables (con una pequeña desviación).

En la distribución de los casos en el Factor 2 (Inteligencia baja-Inteligencia alta), constatamos que un $40 \%$ de los niños puntúan bajo en inteligencia, siendo el pensamiento de estos niños concreto (el 20\% de los niños manifiestan una gran desviación y en el $20 \%$ restante la desviación en este factor de personalidad es pequeña, respectivamente). El $40 \%$ de los niños se sitúan en los valores medios $(\mathrm{Mo}=5)$. Y por último, el 20\% restante puntúan alto en inteligencia (pensamiento abstracto), manifestando una gran desviación.

En la distribución de los casos en el Factor 3 (Emocionalmente afectado - Emocionalmente estable), constatamos que un $60 \%$ de los niños se muestran afectados por los sentimientos y emocionalmente poco estables, manifestando una gran desviación $(\mathrm{Mo}=3)$. Los datos indican que estos niños tienen dificultades para relacionarse con los demás, tienen menos tolerancia a la frustración y son más propensos a perder el control emocional. El 40\% restante de los niños se sitúan en valores medios.

En la distribución de los casos en el Factor 4 (Calmoso - Excitable), constatamos que un $30 \%$ de los niños se muestran calmosos (el 20\% de los niños manifiestan una gran desviación y en el 10\% restante la desviación en este factor de personalidad es pequeña, respectivamente). El 30\% de los niños se sitúan en valores medios. Y por último, el $40 \%$ restante se muestran excitables ante pequeñas provocaciones, mostrándose impacientes y exigentes en determinadas situaciones ( $20 \%$ con una pequeña desviación y $20 \%$ con gran desviación, respectivamente).

En la distribución de los casos en el Factor 5 (Sumiso - Dominante), constatamos que un $60 \%$ de los niños se muestran sumisos, obedientes y dóciles y se acomodan y ceden fácilmente a las diferentes situaciones a las que se enfrentan (el 50\% de los niños manifiestan una gran desviación y en el 10\% restante la desviación en este factor de personalidad es pequeña, 
respectivamente). El 10\% de los niños se sitúan en valores medios. Y por último el $30 \%$ restante se muestran dominantes (obstinados y agresivos), manifestando gran desviación.

En la distribución de los casos en el Factor 6 (Sobrio - Entusiasta), constatamos que un $70 \%$ de los niños puntúan alto en sobriedad (el 60\% de los niños manifiestan una gran desviación y en el 10\% restante la desviación en este factor de personalidad es pequeña, respectivamente). Estos niños se muestran serios y taciturnos. La puntuación alta en sobriedad puede indicar una falta de aprobación del niño respecto a si mismo. El 30\% restante de los niños se sitúan en valores medios.

En la distribución de los casos en el Factor 7 (Despreocupado - Consciente), constatamos que un $50 \%$ de los niños se muestran despreocupados, desatento con las reglas, actuando por conveniencia propia (el $40 \%$ de los niños manifiestan una gran desviación y en el 10\% restante la desviación en este factor de personalidad es pequeña, respectivamente). La alta puntuación parece reflejar la dificultad de los niños para incorporar los valores del mundo de los adultos. El 40\% de los niños se sitúan en valores medios. Y por último el 10\% restante son conscientes (con una pequeña desviación).

A continuación en la tabla 3, vemos los resultados de la aplicación del Cuestionario de Personalidad para niños ESPQ en el resto de los Factores de Primer Orden.

Tabla 3. Resultados del Cuestionario de Personalidad ESPQ en los Factores de Primer Orden (2)

\begin{tabular}{|r|r|r|r|r|r|r|}
\hline DECATIPOS & Factor 8 & Factor 9 & Factor 10 & Factor 11 & Factor 12 & Factor 13 \\
\hline 1 & - & - & - & - & - & - \\
\hline 2 & 2 & - & - & 6 & - & - \\
\hline 3 & 2 & - & - & - & - & - \\
\hline 4 & 10 & - & 2 & - & - & 2 \\
\hline 5 & 4 & 4 & 2 & 4 & - & 2 \\
\hline 6 & 2 & 2 & 2 & 6 & 2 & - \\
\hline 7 & - & 2 & 4 & 4 & 8 & 6 \\
\hline
\end{tabular}




\begin{tabular}{|c|r|r|r|r|r|r|}
\hline 8 & - & 4 & 4 & - & 2 & 10 \\
\hline 9 & - & 8 & 6 & - & 4 & - \\
\hline 10 & - & - & - & - & 4 & - \\
\hline Total & 20 & 20 & 20 & 20 & 20 & 20 \\
\hline
\end{tabular}

Factor 8: Cohibido-Emprendedor Factor 9: Sensibilidad dura-blanda Factor 10: Seguro-Dubitativo

Factor 11: Sencillo-Astuto

Factor 12: Sereno-Aprensivo

Factor 13: Relajado-Tenso

\begin{tabular}{|lccccccc|}
\hline & Media & Sx & Mo & Var & \multicolumn{3}{c|}{$\begin{array}{l}\text { Percentiles } \\
\end{array}$} \\
& & & & & 25 & 50 (Mdn) & 75 \\
\hline Cohibido-emprendedor & 4,100 & 1,101 & 4,0 & 1,211 & 3,750 & 4,000 & 5,000 \\
Sensibilidad dura-blanda & 7,500 & 1,650 & 9,0 & 2,722 & 5,750 & 8,000 & 9,000 \\
Seguro-dubitativo & 7,200 & 1,751 & 9,0 & 3,067 & 5,750 & 7,500 & 9,000 \\
Sencillo-astuto & 4,800 & 2,044 & 2,0 & 4,178 & 2,000 & 5,500 & 6,250 \\
Sereno-aprensivo & 8,000 & 1,414 & 7,0 & 2,000 & 7,000 & 7,500 & 9,250 \\
Relajado-tenso & 7,000 & 1,414 & 8,0 & 2,000 & 6,500 & 7,500 & 8,000 \\
\hline
\end{tabular}

En la distribución de los casos en el Factor 8 (Cohibido - Emprendedor), constatamos que un $70 \%$ de los niños puntúan alto en cohibición (el 20\% de los niños manifiestan una gran desviación y en el 50\% restante la desviación en este factor de personalidad es pequeña, respectivamente). Los datos indican que la mayoría de los niños se muestran tímidos, inhibidos, sensibles, se amedrentan fácilmente y, mediante el alejamiento, intentan evitar la amenaza y excesiva estimulación social. El 30\% restante de los niños se sitúan en valores medios.

En la distribución de los casos en el Factor 9 (Sensibilidad dura - blanda), constatamos que un 70\% de los niños puntúan alto en sensibilidad blanda (el 60\% de los niños manifiestan una gran desviación y en el 10\% restante la desviación en este factor de personalidad es pequeña, respectivamente). Estos niños muestran una mayor dependencia hacia el adulto, sensibilidad fomentada por la sobreprotección. El 30\% de los niños se sitúan en valores me$\operatorname{dios}(\mathrm{Mo}=5)$.

En la distribución de los casos en el Factor 10 (Seguro - Dubitativo), verificamos que un $10 \%$ de los niños se muestran seguros (manifestando una pequeña desviación en este factor de personalidad). El $20 \%$ de los niños se sitúan en valores medios. Y por último el $70 \%$ res- 
tante se muestran dubitativos (20\% con una pequeña desviación y $50 \%$ con gran desviación, respectivamente). La mayoría de los niños se muestran reservados, individualistas, precavidos, reprimidos internamente y no les gusta actuar en grupo.

En la distribución de los casos en el Factor 11 (Sencillo - Astuto), constatamos que un $30 \%$ de los niños puntúan alto en sencillo (llanos, sentimentales y torpes socialmente), el 50\% de los niños se sitúan en valores medios y el $20 \%$ restante se muestran astutos (con una pequeña desviación).

En la distribución de los casos en el Factor 12 (Sereno - Aprensivo), constatamos como dato muy significativo que un $90 \%$ de los niños se muestran aprensivos (el $50 \%$ de los niños manifiestan una gran desviación y en el $40 \%$ restante la desviación en este factor de personalidad es pequeña, respectivamente). La mayoría de los niños se muestran inseguros, irritables, preocupados, ansiosos, con sensación de culpabilidad y autorreproches. El 10\% restante se sitúan en valores medios.

En la distribución de los casos en el Factor 13 (Relajado - Tenso), constatamos que un $10 \%$ de los niños se muestran relajados, manifestando una pequeña desviación en este factor de personalidad. El 10\% de los niños se sitúan en valores medios. Destacar que el $80 \%$ de los niños se muestran tensos (30\% con una pequeña desviación y $50 \%$ con gran desviación, respectivamente). Parecen reflejar un tipo de conducta que hace difícil la sociabilidad (presión, frustración, inquietud, etc.).

A continuación en la tabla 4, vemos los resultados de la aplicación del Cuestionario de Personalidad para niños ESPQ en los Factores de Segundo Orden.

Tabla 4. Resultados del Cuestionario de Personalidad ESPQ en los Factores de Segundo Orden

\begin{tabular}{|c|c|c|}
\hline DECATIPOS & AJUSTE-ANSIEDAD & INTROVERSIÓN-EXTRAVERSIÓN \\
\hline 1 & - & - \\
\hline 2 & - & - \\
\hline
\end{tabular}




\begin{tabular}{|c|c|c|}
\hline 3 & - & 4 \\
\hline 4 & - & 10 \\
\hline 5 & - & 2 \\
\hline 6 & 2 & 2 \\
\hline 7 & 4 & 2 \\
\hline 8 & 10 & - \\
\hline 9 & 4 & - \\
\hline 10 & - & 20 \\
\hline Total & 20 & - \\
\hline
\end{tabular}

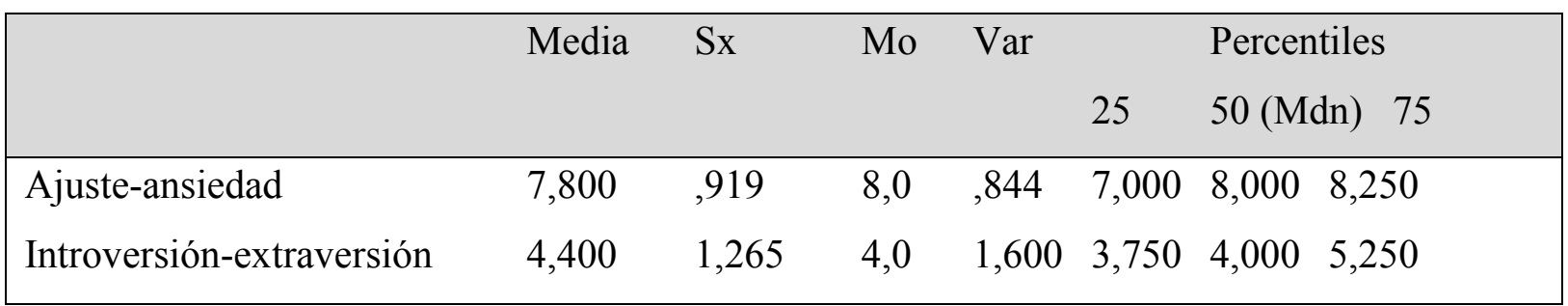

En la distribución de los casos en el Factor 14 (Ajuste - Ansiedad), constatamos como muy significativo que un $90 \%$ de los niños puntúen alto en ansiedad (el $70 \%$ de los niños manifiestan una gran desviación y en el 20\% restante la desviación en este factor de personalidad es pequeña, respectivamente). El 10\% de los niños se sitúan en valores medios.

En la distribución de los casos en el Factor 15 (Introversión - Extraversión), constatamos que un $70 \%$ de los niños se muestran introvertidos (el 20\% de los niños manifiestan una gran desviación y en el 50\% restante la desviación en este factor de segundo orden es pequeña). El 20\% de los niños se sitúan en valores medios. El 10\% restante se muestran extravertidos, presentando una pequeña desviación.

\section{B) Resultados del Cuestionario de Ansiedad infantil (CAS)}

A continuación en la tabla 5 vemos los resultados de la aplicación del Cuestionario de Ansiedad infantil (CAS) en la muestra analizada, constatando como un $80 \%$ de los niños manifiestan ansiedad, de los cuales en el $70 \%$ es elevada. 
Tabla 5. Resultados del Cuestionario de Ansiedad Infantil - CAS

\begin{tabular}{|c|c|}
\hline DECATIPOS & N \\
\hline 1 & - \\
\hline 2 & - \\
\hline 3 & - \\
\hline 4 & 2 \\
\hline 5 & 2 \\
\hline 6 & 2 \\
\hline 7 & 8 \\
\hline 8 & 4 \\
\hline 9 & 2 \\
\hline 10 & 20 \\
\hline Total & \\
\hline
\end{tabular}

\begin{tabular}{|lccccccc|}
\hline & Media & Sx & Mo & Var & \multicolumn{2}{l|}{$\begin{array}{l}\text { Percentiles } \\
\text { (ndn) }\end{array}$} \\
& & & & & 25 & $50(\mathrm{Mdn}$ \\
\hline Ansiedad & 7,800 & 1,476 & 8,0 & 2,178 & 6,750 & 8,000 & 9,000 \\
\hline
\end{tabular}

\section{Discusión}

Los resultados obtenidos en el estudio proporcionan evidencia a favor de las hipótesis formuladas. Se constata que los niños con dislalia infantil analizados tienen dificultades para relacionarse con los demás, se muestran inhibidos, son más propensos a perder el control emocional y manifiestan una ansiedad, tensión e inseguridad superior a la media. Varios de los resultados obtenidos coinciden con los estudios realizados por Solomon (1961), Sussman (1975), Shirkey y Manning (1987), Cantwell y Baker (1987), Perelló (1990), Bruno y Sánchez (1994) y Blood, Ridenour, Dean y Scheffner (2003).

Interpretando los resultados obtenidos del Cuestionario de Personalidad ESPQ y del Cuestionario de Ansiedad Infantil CAS, podemos decir que los niños con dislalia objeto de 
estudio tienen dificultades para relacionarse con los demás, se muestran tímidos, inhibidos, precavidos y reservados en las interacciones sociales (no les gusta actuar en grupo), alejándose del contacto con compañeros. Mediante el alejamiento intentan evitar la amenaza del entorno y la excesiva estimulación social. Los niños se muestran muy sensibles, sensibilidad que puede estar fomentada por la sobreprotección parental. Lo que a su vez puede hacer persistir el desorden articulatorio (Gallardo y Gallego, 1993).

Por otra parte, constatamos que los niños evaluados se amedrentan fácilmente y muestran inestabilidad emocional. Muestran una gran dependencia hacia el adulto (padres y maestros). Tienen menos tolerancia a la frustración y son más propensos a perder el control emocional, manifestando inseguridad, irritabilidad, preocupación y elevados niveles de ansiedad, lo que a su vez hace difícil la sociabilidad. Asimismo, puntúan alto en sobriedad y aprensión, dato que, según Coan y Cattell (2002), indica una falta de aprobación respecto a sí mismo (autorreproches y sentimientos de culpa).

Todo parece indicar que el niño con dislalia, al ser consciente de las dificultades que presenta y del entorno hostil, reacciona mostrando las características de la personalidad mencionadas anteriormente. Por tanto, la dislalia puede afectar sobre el ámbito emocional, personal, social y escolar del niño (Cano y Navarro, 2003). En esta misma línea, autores como Perelló (1990) y Pascual (1998) plantean que en el niño con dislalia puede darse una reacción de frustración, manifestándose bien en forma de agresividad, bien en retraimiento y timidez. El hecho de haber sufrido alguna humillación por esta causa le hará disminuir la confianza en si mismo y generará una inseguridad creciente. Asimismo mantienen la existencia de una relación entre dislalias y timidez, retraimiento y/o apatía.

Tras el análisis exhaustivo de los datos se confirma la tendencia de los niños con dislalia hacia la ansiedad. Este dato se confirma tanto a través del ESPQ como del CAS. Tal y como señalan Gillis y Cattell (1979), el factor de ansiedad de segundo orden aparece configurado a través de los factores de primer orden: excitabilidad, timidez, aprensión, tensión e inestabilidad emocional, factores en los cuales los niños puntúan alto. 
En el artículo hemos podido comprobar la enorme cantidad de características psicológicas asociadas a una de las patologías de mayor incidencia en la población escolar durante las etapas iniciales. Constatamos la dificultad existente a la hora de delimitar si los rasgos de personalidad de estos niños causan el desorden lingüístico, o en cambio son un efecto del mismo. Hay que destacar el importante papel que desempeña la familia en la instauración y/o mantenimiento de patrones de personalidad disfuncionales y la influencia nefasta de un entorno social y escolar poco adaptativo sobre estos niños.

Es necesario conocer la influencia del entorno familiar en la instauración de un patrón de personalidad específico que predisponga al niño a una dificultad lingüística determinada. Y es preciso determinar si existen diferencias en la personalidad del niño según el momento de instauración del trastorno del lenguaje. Es decir, antes del inicio de la patología, cuando se inician las dificultades y cuando el cuadro ya se ha implantado. Esto proporcionará un mayor conocimiento sobre las implicaciones de la personalidad en la dislalia infantil.

Por otra parte, debemos tener en cuenta las actitudes y reacciones del niño con dislalia frente al entorno familiar, social y escolar. Puede que el niño se sienta objetivamente marginado como consecuencia de su dificultad articulatoria, o puede que sea él mismo el que se margine aislándose de los demás como consecuencia de su desvalorización. Así el niño, evitando el contacto con cualquier persona de su entorno más o menos cercano, consigue reducir el malestar que le genera la interacción comunicativa.

Una de las características comunes en los niños con dislalia, que puede condicionar en cierto modo su personalidad, es la toma de conciencia del problema. No es fácil relacionarse con los demás cuando uno tiene conciencia de sus limitaciones. Es normal que el niño en cada situación comunicativa tenga dudas acerca de su eficacia. Esto provoca en él una fuerte inseguridad, que merma aún más su patrón de habla. La toma de conciencia del problema puede repercutir en su rendimiento académico, ya que algunos de estos niños presentan serias dificultades a la hora de concentrarse en tareas escolares, tendiendo a mostrarse con mucha frecuencia distraídos y ausentes. 
Algunos niños al ser conscientes de sus dificultades articulatorias tienden a cohibirse a la hora de hablar en el aula escolar y en sus interacciones con compañeros y adultos, todo ello por temor a ser objeto de burlas, risas y críticas. Esto, a su vez, puede elevar la tensión en los músculos implicados en la articulación, agravando aún más la dificultad que presentan.

En la dislalia, al igual que en otras patologías, es importante explorar características personales del niño tales como: los signos de desvalorización, las conductas agresivas hacia el entorno y hacia sí mismo, los comportamientos regresivos, el grado de satisfacción personal, familiar y social, la actitud y el ajuste emocional, el grado de conflictividad parental y fraternal, los sentimientos de culpa, el grado de afectividad que reciben a nivel familiar, la actitud interpersonal, etc.

En el estudio hemos podido constatar que determinadas características de la personalidad del niño (inmadurez, inestabilidad emocional, etc.) y determinadas circunstancias familiares, sociales y escolares pueden favorecer la aparición de la dislalia. De igual manera, comprobamos que muchos de los rasgos de personalidad que manifiestan estos niños son más una consecuencia del trastorno del habla que un factor predisponente al mismo (timidez, ansiedad, temor a hablar, agresividad, reducción de las interacciones sociales, aislamiento social, etc.)

Delimitar las características de la personalidad del niño con dislalia nos permitirá adaptarnos a su realidad durante el proceso de intervención. Debemos tener muy presentes los aspectos personales, relacionales, sociales y emocionales del niño, dado que el conocimiento de los mismos, puede facilitar la superación más rápida de las dificultades que presenta. 


\section{Referencias}

Aguilar, A. (1991). Taxonomía de los trastornos del lenguaje. En A. Aguilar, Psicopatología del lenguaje I. Un modelo integral del intervención (pp. 73-79). Barcelona: PPU.

Ajuriaguerra, J. y Marcelli, D. (1982). Psicopatología del lenguaje. En J. Ajuriaguerra y D. Marcelli, Manual de Psicopatología del niño (pp. 96-111). Barcelona: Toray-Masson.

Blood, G. W., Ridenour, V.J., Dean, C. y Scheffner, H. (2003). Co-occurring disorders in children who stutter. Journal of Communication Disorders, 36(6), 427-448.

Bruno, C. y Sánchez, M. (1994). Dislalias. En J. Peña-Casanova, Manual de logopedia, (2a Ed.) (pp. 153 - 172). Barcelona: Masson..

Busto, M. C. (1998). Alteraciones del lenguaje oral. En M.C. Busto, Manual de logopedia escolar. Niños con alteraciones del lenguaje oral en Educación Infantil y Primaria (pp. 83-110). Madrid: CEPE.

Cano, M.I. y Navarro, M.I. (2003). Dificultades en el desarrollo del habla y del lenguaje oral en la infancia y la adolescencia. En M. Puyuelo y J.A. Rondal, Manual de desarrollo y alteraciones del lenguaje (pp. 323 - 367). Barcelona: Masson.

Cantwell, D. P. y Baker, L. (1987). The prevalence of anxiety in children with communication disorders. Journal of Anxiety Disorders, 1(3), 239-248.

Coan, R. W. y Cattell, R. B. (2002). Cuestionario de personalidad para niños ESPQ. Madrid: TEA.

Gallardo, J.R. y Gallego, J.L. (1993). Alteraciones de la articulación: dislalias. En J.R. Ga1lardo y J.L. Gallego, Manual de Logopedia Escolar. Un enfoque práctico (pp.171220). Málaga: Aljibe.

García-Valdecasas, M. (2000). Trastornos más usuales en el ámbito educativo. En Peñafiel, F. y Fernández, J.D. (Coords.), Cómo intervenir en logopedia escolar. Resolución de casos prácticos (pp. 150-166). Madrid: CCS.

Gillis, J. S. (1999). Cuestionario de Ansiedad Infantil CAS. Madrid: TEA.

Gillis, J. S. y Cattell, R. B. (1979). Comparison of second order personality structures at 6-8 year with later patterns. Multivariate Ex-Clinic Research, 4, 93-99.

Massana, M. y Artal, M. (1997). Dislalias. En M. Puyuelo y cols., Casos clínicos en logopedia (pp. 215 - 243). Barcelona: Masson. 
Moreno, J.M. (2004). Características de la personalidad y alteraciones del lenguaje en Educación Infantil y Primaria. Revista Iberoamericana de Educación, 34(1), 1-12.

Pascual, P. (1998). Incidencia recíproca entre el habla y la personalidad. En P. Pascual, La dislalia. Naturaleza. Diagnóstico y Tratamiento (10ª Ed.) (pp. 45-47). Madrid: CEPE.

Perelló, J. (1990). Dislalia. En J. Perelló, Trastornos del habla (pp. 283-348). Barcelona: Masson.

Ramos, F. y Manga D. (1996). Psicopatología del lenguaje. En A. Belloch, B. Sandin y F. Ramos, Manual de Psicopatología Vol. 1 (pp. 336-378). Madrid: McGraw-Hill.

Serón, J.M. y Aguilar, M. (1992). Los problemas del habla en el niño. En J.M. Serón y M. Aguilar, Psicopedagogía de la comunicación y el lenguaje (pp. 279-321). Madrid: EOS.

Shirkey, E. A. y Manning, W.H. (1987). The effects of emotional arousal and increased speaking rate on children's newly learned /r/ productions. Journal of Communications Disorders, 20(1), 51-60.

Solomon, A. (1961). Personality and behaviour patterns of children with functional defects of articulation. Child Development, 32(4), 731-737

Sussman, H.M. (1975). Hemispheric specialization for speech production and perception in stutterers. Neuropsychologia, 13, 19-26.

Waller, M., Sollod, R., Sander, E., Kunkicki, E. (1983). Psychological assessment of speech and language-disordered children. Language, Speech and Hearing Services in the Schools, 14 (2), 92-98. 